\title{
Mild Decrease in Skin Temperature Reduces the Heart Rate during Moderate Exercise
}

\author{
Masayuki Konishi ${ }^{1}$, Hiroki Tabata ${ }^{2,3}$, Karina Ando $^{4}$, Hyeon-Ki Kim ${ }^{3}$, Mio Nishimaki ${ }^{2,4}$, Mi Xiang ${ }^{2}$ and Shizuo \\ Sakamoto ${ }^{1}$ \\ 1. Faculty of Sport Sciences, Waseda University, Tokorozawa, Saitama 359-1192, Japan \\ 2. Graduate School of Sport Sciences, Waseda University, Tokorozawa, Saitama 359-1192, Japan \\ 3. Research Fellow of the Japan Society for the Promotion of Science, Chiyoda-ku, Tokyo 102-0083, Japan \\ 4. Japan Institute of Sports Sciences, Kita-ku, Tokyo 115-0056, Japan
}

\begin{abstract}
The purpose of this study was to investigate the effects of a mild decrease in skin temperature on the heart rate, substrate oxidation, and hormonal responses during exercise. Ten young men performed treadmill exercises approximately $65 \%$ of their maximal oxygen uptake for $60 \mathrm{~min}$ under two trials: (1) mild body cooling trial, and (2) control trial. Rectal and skin temperatures were recorded during and after exercise. Heart rate and oxygen uptake were monitored during exercise. Blood samples for catecholamine, cortisol, free fatty acid, insulin, and glucose concentrations were collected before, immediately after, and $1 \mathrm{~h}$ after exercise. In the mild body cooling trial, mean skin temperature decreased by $1.7 \pm 0.7^{\circ} \mathrm{C}$ in the last minutes of exercise, compared with before exercise. No differences in rectal temperature were seen between the two trials. Although oxygen uptake during exercise and blood parameters did not differ between the trials, heart rate and subjective fatigue during exercise was lower in the mild body cooling trial than in the control trial. This study suggests that mild body cooling reduces the heart rate and subjective fatigue during moderate exercise, but does not affect substrate oxidation or hormonal responses to exercise.
\end{abstract}

Key words: Mild body cooling, climatic terrain therapy, cold exposure.

\section{Introduction}

Climatic terrain therapy can promote health and is covered by insurance in Germany. Furthermore, the positive effects of climatic terrain therapy have been demonstrated in patients with functional cardio-circulatory disorders [1]. To obtain physiological effects, participants undergo climatic terrain therapy daily over several weeks. Climatic terrain treatment involves endurance training, which improves heart capacity, blood circulation, lung function, and muscular metabolism [2]. The endurance training involves walking on a sloping terrain with mild body cooling (a decrease of approximately $2^{\circ} \mathrm{C}$ in skin temperature) [2]. The cold stimulus is a critical element; it leads to physiological changes that promote health

Corresponding author: Masayuki Konishi, Ph.D., assistant professor, research field: exercise physiology.
[2]. Although it is thought that mild cooling during climatic terrain therapy leads to adaptations in the skin, muscles, circulation, and metabolism, the physiological mechanisms are not fully understood [2]. For evidence-based healthcare to utilize climatic terrain therapy, it is important to investigate the mechanisms underlying the effects of mild body cooling on physiological parameters.

In contrast to mild body cooling, the effects of severe body cooling on certain physiological parameters are well documented. Exposure to severe cold with a decrease in rectal and skin temperatures enhances fat oxidation during running and walking [3]. In addition, participants exposed to cold who were shivering had increased carbohydrate utilization during walking at low intensity compared to those who were not shivering [4]. In contrast, lipid and carbohydrate oxidation during submaximal exercise did not differ 
between a mild cooling environment $\left(10^{\circ} \mathrm{C}\right)$ and a neutral environment $\left(20^{\circ} \mathrm{C}\right)$ [5]. Despite differences in the degree of cold exposure, researchers have consistently observed heart rate reductions during exercise in a cold environment, compared to that during exercise in a neutral environment [3-6].

Typically, researchers examining the effect of exposure to the cold on physiological parameters have used a decrease in ambient temperature as the cold stimulus and have observed reductions in skin temperature of more than $5^{\circ} \mathrm{C}$ [3-5]. In addition, a previous study examined the effects of a $1.5^{\circ} \mathrm{C}$-decrease in skin temperature using evaporative cooling shirts on peripheral blood flow at rest, but did not examine the effects during exercise [7]. Thus, to our knowledge, no study has focused the effects of a $2{ }^{\circ} \mathrm{C}$-decrease in skin temperature, a common characteristic of climatic terrain therapy, on metabolism or heart rate during exercise.

Heart rate during exercise is a useful marker for indicating exercise intensity in participants who are training or are prescribed exercise [8]. To better control exercise intensity in climatic terrain therapy using heart rate, a clear understanding of the effects of mild body cooling on heart rate during exercise is needed. Therefore, the purpose of the present study was to investigate the effects of a mild decrease in skin temperature on heart rate and metabolism during exercise. We hypothesized that mild body cooling at room temperature would decrease the heart rate during exercise, but would not influence metabolism.

\section{Methods}

\subsection{Participants}

Ten young men (age, $27 \pm 3$ years; height, $172.4 \pm$ $7.9 \mathrm{~cm}$; body mass, $67.5 \pm 9.2 \mathrm{~kg}$; body fat, $16.6 \pm 5.8 \%$ ) (mean $\pm \mathrm{SD}$ ) participated. All participants were free of any major illnesses and were not currently taking any medications or supplements. The participants received a verbal and written description of the study and provided informed consent. This study followed the
Declaration of Helsinki and was approved by the institutional ethics committee of Waseda University (2013-246).

\subsection{Preliminary Session}

Before the main experimental trials, participants performed the Bruce treadmill test on a motor-driven treadmill (MAT-2700, Fukuda Denshi, Japan) to determine peak oxygen uptake $\left(\dot{\mathrm{VO}}_{2}\right.$ peak) [9]. Participants were required to abstain from alcohol and avoid intense physical activity 1 day prior to the preliminary test. Participants ate lunch $4 \mathrm{~h}$ before the test, and consumed water ad libitum until the start of the test. During the test, a 12-lead electrocardiogram was electronically recorded (STRESS TEST SYSTEM ML-9000, FUKUDA DENSHI, Japan) and the heart rate was derived from the R-R interval. Pulmonary gas exchange, including oxygen uptake $\left(\dot{\mathrm{V}}_{2}\right)$, carbon dioxide output $\left(\mathrm{VCO}_{2}\right)$, minute ventilation (VE), and respiratory exchange ratio (RER) were determined breath-by-breath using a gas analyser (AE-300 S, MINATO MEDICAL SCIENCE, Japan). Participants were considered to have reached maximal effort if they demonstrated a plateau in $\dot{\mathrm{VO}}_{2}$, defined as a change in $\mathrm{VO}_{2}<2.1 \mathrm{~mL} / \mathrm{kg} / \mathrm{min}$ over the final successive stages of the test, or if at least 2 of the following secondary criteria were achieved: the highest heart rate reached $95 \%$ of the age-predicted maximal heart rate (220-age), the highest RER was $>1.05$, and the participant demonstrated clear subjective symptoms of fatigue [10]. The highest recorded 30-s moving average of the $\mathrm{VO}_{2}$ during the exercise test was recorded as the $\mathrm{VO}_{2}$ peak.

\subsection{Experimental Protocols}

Each participant performed the treadmill exercise at approximately $65 \%$ of their $\dot{\mathrm{VO}}_{2}$ peak for 60 minutes during two trials: (1) a mild body cooling trial, and (2) a control trial. The trials were completed with a 1-week interval between trials using a randomized cross-over design. As in the preliminary test, participants were 
required to abstain from alcohol and avoid intense physical activity 1 day before the trials. The participants ate an identical provided breakfast before $0900 \mathrm{~h}$ on trial days, and consumed water ad libitum until the start of the trial. All participants arrived in the laboratory at $1300 \mathrm{~h}$. The laboratory was maintained at an ambient temperature of $25^{\circ} \mathrm{C}$ and $50 \%$ relative humidity. Body mass and body fat were measured using a digital scale with impedance (InnerScan, BC-520, TANITA, Japan) to the nearest $0.1 \mathrm{~kg}$. Height was measured using a stadiometer to the nearest 0.1 $\mathrm{cm}$.

In the mild body cooling trial, the participants wore identical half-sleeved t-shirts and shorts provided by us, and were sprayed with cold water at approximately $10^{\circ} \mathrm{C}$ using a sprayer with a fan $(1.5-2.0 \mathrm{~m} / \mathrm{s})$ during exercise. We sprayed their arms and legs from the front approximately every $5 \mathrm{~min}$; patients in climatic terrain therapy actually cool their arms and legs. The frequency of sprayer was controlled depending on the skin temperature, was assessed every 5 minutes. The frequency of the sprayer was increased if the skin temperatures did not drop by $2^{\circ} \mathrm{C}$. In the control trial, the participants wore the same provided clothes and performed the exercise without being sprayed.

Before the exercise began, participants were asked to self-insert a rectal probe. Skin temperature thermistors, electrocardiogram electrodes, and a mask for gas analysis were also attached. Participants rested in a seated position for $20 \mathrm{~min}$ to acclimate to the ambient temperature; during this time the participants were instrumented. Heart rate and breath-by-breath measurements were recorded for $5 \mathrm{~min}$, with the average of the last $1 \mathrm{~min}$ used to reflecting resting values.

The treadmill speed and slope (NT-25, NISHIKAWA IRON WORKS, Japan) required to achieve $65 \%$ of the $\dot{\mathrm{VO}}_{2}$ peak was derived from the participant's preliminary exercise test, and was adjusted according to the oxygen uptake within $10 \mathrm{~min}$ from the beginning of the exercise. Heart rate and breath-by-breath measurements were recorded throughout the exercise, and were averaged over $5 \mathrm{~min}$ every 10 min. Pulmonary gas exchange $\left(\dot{\mathrm{VO}}_{2}, \dot{\mathrm{V}}_{2}\right.$, and RER) were determined breath-by-breath using a gas analyser (AE-310 S, MINATO MEDICAL SCIENCE, Japan). Fat and carbohydrate (CHO) oxidation rates were determined from $\mathrm{VO}_{2}$ and $\dot{\mathrm{VCO}}_{2}$ values using the following equation [11]:

Fat oxidation $(\mathrm{g} / \mathrm{min})=1.67 \times \mathrm{VO}_{2}(\mathrm{~L} / \mathrm{min})-1.67$ $\times \mathrm{VCO}_{2}(\mathrm{~L} / \mathrm{min})$

$\mathrm{CHO}$ oxidation $(\mathrm{g} / \mathrm{min})=4.55 \times \dot{\mathrm{V} C \mathrm{O}_{2}}(\mathrm{~L} / \mathrm{min})-$ $3.21 \times \mathrm{VO}_{2}(\mathrm{~L} / \mathrm{min})$

This equation assumes a negligible urinary nitrogen excretion rate and $\mathrm{CO}_{2}$ production from lactic acid buffering. In addition, the rating of perceived exertion (RPE) was assessed every 10 min during the exercise. Blood samples for determining epinephrine, norepinephrine, cortisol, free fatty acid (FFA), insulin, and glucose concentrations were collected before, immediately after, and $1 \mathrm{~h}$ after exercise.

\subsection{Rectal and Skin Temperature Measurements}

Rectal temperature $\left(\mathrm{T}_{\mathrm{re}}\right)$ was measured using a rectal thermistor (LT-ST08-11, Gram Corporation, Japan) inserted $10 \mathrm{~cm}$ beyond the anal sphincter. Skin temperatures were measured at 4 sites using surface thermistors (LT-ST08-12, Gram Corporation, Japan) attached with a single layer of waterproof tape; the $\mathrm{T}_{\mathrm{sk}}$ was calculated using the following equation [12]:

$T_{\text {sk }}=0.3$ (chest) +0.3 (upper arm) +0.2 (thigh) + 0.2 (shin)

Rectal and skin temperatures were recorded at rest and during exercise every $30 \mathrm{~s}$ using a data logger (LT-8, Gram Corporation, Japan). The data at every 10 min were used for analysis.

\subsection{Blood Sampling and Analyses}

Samples for analysis of plasma epinephrine, norepinephrine, and cortisol concentrations were placed in 7-mL tubes containing EDTA. Serum samples for the analysis of insulin and FFA 
concentrations were placed in 9-mL tubes containing thrombin. Samples for the analysis of plasma glucose concentrations were placed in 2-mL tubes containing sodium fluoride, heparin, and EDTA- 2Na. All sets of tubes were centrifuged at $3000 \mathrm{rpm}$ for $10 \mathrm{~min}$ after collection, after which the serum and plasma were transferred into plastic tubes and then immediately stored at $-80^{\circ} \mathrm{C}$ until further analysis. Plasma epinephrine and norepinephrine concentrations were measured using high-performance liquid chromatography. Serum insulin concentrations were measured using a chemiluminescent enzyme immunoassay (CLEIA). Serum FFA concentrations were measured using enzymatic assays. Plasma glucose concentrations were measured via the hexokinase/UV method. All blood samples were analysed by SRL, Inc. (Tokyo, Japan).

\subsection{Statistical Analysis}

All data were expressed as mean $\pm \mathrm{SD}$. A two-way analysis of variance for repeated measures (trial $\times$ time) with Bonferroni post-hoc analysis was used to compare heart rate, oxygen uptake, RER, fat and glucose oxidation, RPE, skin and rectal temperatures, and blood parameters. Greenhouse-Geisser corrections were applied, where appropriate, and only adjusted results are reported. Statistical analyses were performed using SPSS Statistics 21.0 software (IBM, Tokyo, Japan). $P$ values $<0.05$ were considered significant.

\section{Results}

The ambient temperature and humidity did not differ between trails (at baseline: mild body cooling trial, $25.0 \pm 0.3^{\circ} \mathrm{C}, 49.8 \pm 3.5 \%$; control trial, $24.9 \pm 0.3^{\circ} \mathrm{C}$, $49.6 \pm 3.7 \%$; during exercise: mild body cooling trial, $24.9 \pm 0.2^{\circ} \mathrm{C}, 50.9 \pm 2.4 \%$; control trial, $25.0 \pm 0.2^{\circ} \mathrm{C}$, $50.0 \pm 3.1 \%$, respectively). Rectal $\left(\mathrm{T}_{\mathrm{re}}\right)$ and skin temperatures during exercise are presented in Fig. 1. $\mathrm{T}_{\mathrm{re}}$ increased over time $(P<0.01)$, but did not differ between trials. In the last minutes of the exercise during the mild body cooling trial, skin temperature decreased by $1.7 \pm 0.7^{\circ} \mathrm{C}$ compared to that before exercise. A significant interaction between trial and time was observed for $\mathrm{T}_{\mathrm{sk}}(P<0.01)$. Post-hoc analyses showed that the $\mathrm{T}_{\mathrm{sk}}$ was lower in the mild body cooling trial compared to that in the control trial except at rest $(P<$ 0.001 at $10,20,30,40,50$, and $60 \mathrm{~min}$, respectively).

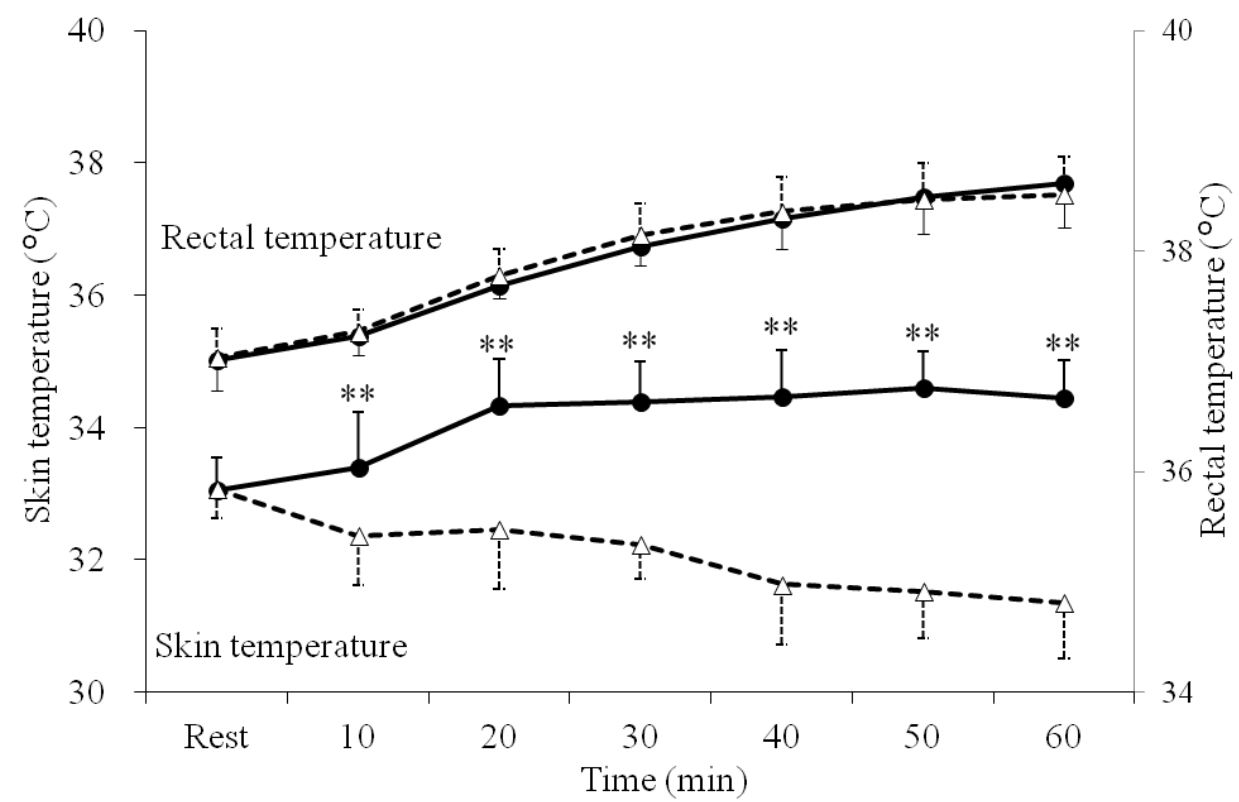

Fig. 1 Rectal and skin temperatures at rest and during 60 min of exercise in the mild body cooling $(\Delta)$ and control $(\bullet)$ trials. Significant difference between the two trials: $* * P<0.01$. 
The $\mathrm{VO}_{2}$ peak, RER, fat oxidation, $\mathrm{CHO}$ oxidation, and RPE at rest and during exercise are shown in Table 1. The $\dot{\mathrm{VO}}_{2}$ peak was $49.3 \pm 6.6 \mathrm{~mL} / \mathrm{kg} / \mathrm{min}$. The mean values of $\% \mathrm{VO}_{2}$ peak during the 60 -min exercise period were $65.3 \pm 4.5 \%$ and $66.5 \pm 5.0 \%$ for the mild body cooling and control trials, respectively. No differences in $\mathrm{VO}_{2}$ values at rest or during exercise were observed between the trials (Table 1). A significant trial $\times$ time interaction was observed for the RER $(P<0.05)$. Post-hoc analyses revealed that the RER at rest was higher in the mild body cooling trial compared to that in the control trial $(P<0.05)$; in contrast, the RER during exercise did not differ between the trials. Fat oxidation during exercise increased over time (main effect of time: $P<0.01$ ), whereas $\mathrm{CHO}$ oxidation during exercise decreased over time (main effect of time: $P<0.01$ ); however, fat and $\mathrm{CHO}$ oxidation did not differ between the trials. There was a significant trial $\times$ time interaction for the RPE $(P<0.01)$. Post-hoc analyses showed that the RPE at $60 \mathrm{~min}$ of exercise was lower in the mild body cooling trial compared to that in the control trial $(P<0.05)$. Moreover, a significant interaction between trial and time was observed for HR $(P<0.01)$. Post-hoc analyses showed that the HR at 20,30,40,50, and 60 min of exercise was significantly lower in the mild body cooling trial compared to that in the control trial $(P<0.05, P<0.01$, $P<0.01, P<0.01$, and $P<0.01$, respectively, Fig. 2).
Table 2 presents the plasma epinephrine, norepinephrine, and cortisol concentrations, as well as serum insulin and FFA concentrations at rest, immediately after exercise, and $1 \mathrm{~h}$ after exercise. The insulin data of one participant were excluded because the measurement was below $0.3 \mu \mathrm{U} / \mathrm{mL}$ at $1 \mathrm{~h}$ after exercise. A main effect of time was observed for plasma epinephrine $(P<0.01)$, norepinephrine $(P<$ $0.01)$, and serum insulin $(P<0.01)$; however, no trial $\times$ time interactions were detected. There was no significant difference in plasma cortisol concentration between the trials. Plasma glucose concentrations were lower in the mild body cooling trial compared to that in the control trial $(P<0.05)$; however, no significant interaction was observed. Although a trial $\times$ time interaction for serum FFA concentrations was significant $(P<0.05)$, the post-hoc analyses did not detect significant differences between the trials.

\section{Discussion}

The present study is unique because, to the best of our knowledge, it is the first to investigate the effect of a mild decrease in skin temperature using a cold-water spray and fan on heart rate and metabolism during exercise. Our main finding was that mild body cooling reduced heart rate and subjective fatigue during exercise, compared to that during the control condition, even though the participants had the same exercise

Table 1 The oxygen uptake $\left(\mathrm{VO}_{2}\right)$, the respiratory exchange ratio (RER), fat oxidation, carbohydrate oxidation (CHO) and ratings of perceived exertion (RPE) at rest and during $60 \mathrm{~min}$ of exercise in the mild body cooling and control trials.

\begin{tabular}{|c|c|c|c|c|c|c|c|c|}
\hline & & Rest & $10 \mathrm{~min}$ & $20 \mathrm{~min}$ & $30 \mathrm{~min}$ & $40 \mathrm{~min}$ & $50 \mathrm{~min}$ & $60 \mathrm{~min}$ \\
\hline \multirow{2}{*}{$\begin{array}{l}\mathrm{VO}_{2} \\
(\mathrm{~mL} / \mathrm{kg} / \mathrm{min})\end{array}$} & Mild body cooling & $3.6 \pm 0.4$ & $32.7 \pm 3.9$ & $31.6 \pm 3.6$ & $31.7 \pm 4.0$ & $32.0 \pm 4.2$ & $32.4 \pm 4.3$ & $32.3 \pm 4.5$ \\
\hline & Control & $3.8 \pm 0.6$ & $32.8 \pm 3.5$ & $32.1 \pm 3.5$ & $32.1 \pm 4.3$ & $32.6 \pm 4.2$ & $32.9 \pm 4.4$ & $33.3 \pm 4.7$ \\
\hline \multirow{2}{*}{ RER } & Mild body cooling & $0.79 \pm 0.04 *$ & $0.91 \pm 0.03$ & $0.89 \pm 0.03$ & $0.88 \pm 0.03$ & $0.87 \pm 0.03$ & $0.86 \pm 0.03$ & $0.85 \pm 0.03$ \\
\hline & Control & $0.73 \pm 0.07$ & $0.90 \pm 0.04$ & $0.88 \pm 0.05$ & $0.87 \pm 0.05$ & $0.86 \pm 0.05$ & $0.86 \pm 0.04$ & $0.85 \pm 0.04$ \\
\hline \multirow{2}{*}{$\begin{array}{l}\text { Fat oxidation } \\
(\mathrm{g} / \mathrm{min})\end{array}$} & Mild body cooling & $0.09 \pm 0.02$ & $0.33 \pm 0.12$ & $0.41 \pm 0.09$ & $0.46 \pm 0.10$ & $0.50 \pm 0.10$ & $0.56 \pm 0.11$ & $0.59 \pm 0.11$ \\
\hline & Control & $0.12 \pm 0.03$ & $0.37 \pm 0.13$ & $0.44 \pm 0.13$ & $0.48 \pm 0.15$ & $0.53 \pm 0.15$ & $0.55 \pm 0.12$ & $0.60 \pm 0.11$ \\
\hline \multirow{2}{*}{$\begin{array}{l}\text { CHO oxidation } \\
(\mathrm{g} / \mathrm{min})\end{array}$} & Mild body cooling & $0.09 \pm 0.05$ & $2.05 \pm 0.62$ & $1.78 \pm 0.69$ & $1.66 \pm 0.60$ & $1.59 \pm 0.61$ & $1.49 \pm 0.55$ & $1.41 \pm 0.55$ \\
\hline & Control & $0.06 \pm 0.04$ & $1.99 \pm 0.71$ & $1.75 \pm 0.78$ & $1.66 \pm 0.83$ & $1.58 \pm 0.81$ & $1.54 \pm 0.76$ & $1.45 \pm 0.75$ \\
\hline \multirow{2}{*}{ RPE } & Mild body cooling & $6.3 \pm 0.7$ & $10.9 \pm 2.3$ & $11.6 \pm 2.2$ & $12.1 \pm 2.6$ & $12.2 \pm 2.7$ & $12.4 \pm 3.0$ & $12.3 \pm 2.9^{*}$ \\
\hline & Control & $6.3 \pm 0.9$ & $10.7 \pm 2.2$ & $11.8 \pm 2.1$ & $12.1 \pm 2.1$ & $13.1 \pm 2.1$ & $13.6 \pm 2.2$ & $14.3 \pm 2.9$ \\
\hline
\end{tabular}

Values are mean \pm SD. Significant difference between the two trials: $* P<0.05$ 


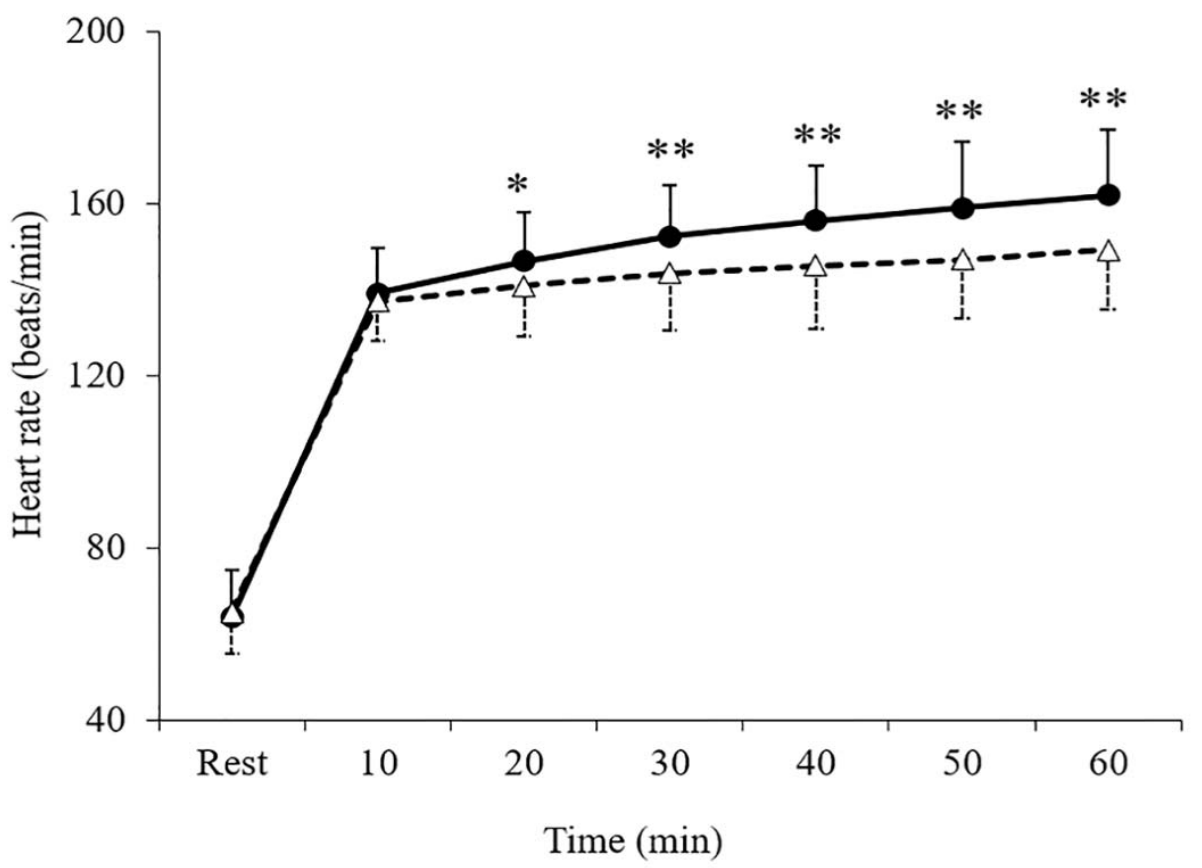

Fig. 2 Heart rate at rest and during $60 \mathrm{~min}$ of exercise in the mild body cooling $(\Delta)$ and control $(\bullet)$ trials. Significant difference between the two trials: $* P<0.05, * * P<0.01$.

Table 2 Epinephrine, norepinephrine, cortisol, insulin, glucose and free fatty acid concentrations at rest, immediately after exercise and $1 \mathrm{~h}$ after exercise in the mild body cooling and control trials.

\begin{tabular}{lllllll}
\hline & \multicolumn{3}{c}{ Mild body cooling } & \multicolumn{2}{c}{ Control } \\
\cline { 2 - 7 } & Rest & $\begin{array}{l}\text { Immediately } \\
\text { after exercise }\end{array}$ & $\begin{array}{l}\text { 1 h after } \\
\text { exercise }\end{array}$ & Rest & $\begin{array}{l}\text { Immediately } \\
\text { after exercise }\end{array}$ & $1 \mathrm{~h}$ after exercise \\
\hline Epinephrine $(\mathrm{pg} / \mathrm{mL})$ & $43.4 \pm 20.6$ & $113.4 \pm 46.2$ & $32.2 \pm 13.4$ & $40.8 \pm 22.5$ & $98.7 \pm 41.1$ & $32.8 \pm 15.2$ \\
Norepinephrine $(\mathrm{pg} / \mathrm{mL})$ & $277.7 \pm 75.4$ & $789.1 \pm 273.4$ & $329.1 \pm 115.1$ & $279.4 \pm 84.5$ & $961.6 \pm 554.4$ & $361.5 \pm 144.4$ \\
Cortisol $(\mu \mathrm{g} / \mathrm{dL})$ & $10.8 \pm 2.7$ & $11.7 \pm 3.3$ & $12.0 \pm 4.9$ & $10.8 \pm 4.3$ & $13.0 \pm 6.4$ & $13.8 \pm 7.6$ \\
Insulin $(\mu \mathrm{U} / \mathrm{mL})$ & $4.6 \pm 3.1$ & $1.5 \pm 1.7$ & $2.4 \pm 0.6$ & $4.4 \pm 2.2$ & $2.3 \pm 1.4$ & $3.4 \pm 2.1$ \\
Glucose $(\mathrm{mg} / \mathrm{dL})$ & $91.7 \pm 5.8$ & $85.7 \pm 4.5$ & $88.3 \pm 2.9$ & $94.0 \pm 6.4$ & $94.0 \pm 10.2$ & $91.6 \pm 3.7$ \\
Free fatty acid $(\mu \mathrm{Eq} / \mathrm{L})$ & $387.3 \pm 111.7$ & $807.6 \pm 279.9$ & $668.2 \pm 111.2$ & $505.9 \pm 217.7$ & $985.1 \pm 346.8$ & $641.0 \pm 217.3$ \\
\hline
\end{tabular}

Values are mean \pm SD.

intensity. However, a mild decrease in skin temperature did not affect fat and $\mathrm{CHO}$ oxidation or hormonal responses during exercise.

In the present study, we used a spray bottle and fan to reduce the skin temperature by approximately $2^{\circ} \mathrm{C}$, which is similar to the reduction seen in climatic terrain therapy. Previous studies examining the effects of exposure to cold on physiological parameters have typically decreased ambient temperature as a method for exposure to cold [3-6]. In the present study, the ambient temperature and relative humidity were the same in the mild body cooling and control trials. Furthermore, by using a spray bottle and fan to reduce the skin temperature, we could investigate the effect of a mild decrease in skin temperature on physiological parameters without a decrease in rectal temperature, allowing the experimental creation of a climatic terrain treatment.

The present results suggest that a mild decrease in skin temperature reduces heart rate during exercise. The physiological demand was comparable between the two trials, as no differences in $\mathrm{VO}_{2}$ were observed during exercise. A previous study showed that skin cooling induces a reduction in peripheral blood flow following peripheral vasoconstriction [7]. Redirecting peripheral blood flow to the core leads to an increase in 
central blood volume and stroke volume [13]. Furthermore, the lack of a difference in $\mathrm{VO}_{2}$ during exercise indicates that cardiac output during exercise in the mild body cooling trial may be similar to that in the control environment. As a result, heart rate during exercise may have been reduced by mild body cooling via an increase in stroke volume. In another study, researchers found that trigeminal nerve stimulation using cold sprays and fan on the face was associated with a reduction in heart rate during exercise [14]. Although these mechanisms should be further investigated by measuring skin blood flow and stroke volume, our current results suggest that even mild body cooling reduces heart rate during exercise, similar to previously published data [3-6].

The results of this study indicate that the RPE and heart rate were decreased by mild body cooling, as compared with those in the control condition. In a previous study, researchers investigated the RPE during exercise in hot and cool conditions and found a lower RPE and skin temperature in the cool condition compared to that in the hot condition [15]. The authors reported that skin temperature, rather than core temperature, was a contributor to effort perception [15]. Similarly, in the study a decrease in skin temperature without a reduction in core temperature was observed. Thus, the observed reduction in the RPE may have been induced by a decrease in skin temperature with a perception of comfort. Furthermore, a previous study reported that it is difficult to accurately control exercise intensity in a cool condition using the RPE [15]. Therefore, a practical application of our results is that, in an exercise prescription for the improvement of cardiovascular disease using mild body cooling, the RPE should be treated with caution in order not to exceed the adequate exercise intensity. The RPE in the mild body cooling trial was lower compared to that in the control trial only at $60 \mathrm{~min}$ of exercise. The effects of mild body cooling on the RPE may be small because the reduction of skin temperature during the first 30 min of exercise was less than $1^{\circ} \mathrm{C}$.
In the present study, mild body cooling did not affect substrate oxidation during exercise or hormonal responses to exercise. Exposure to severe cold with a decrease in rectal and skin temperatures has been shown to enhance fat oxidation during running and walking [3] while lipid and carbohydrate oxidation during submaximal exercise does not differ between mild cooling and neutral environments [5]. In our study, skin temperature was decreased by mild body cooling, while the rectal temperature did not differ between the two trials. The results of a previous study suggest that fuel oxidation in the cold environment is related to a decrease in skin and rectal temperatures, whereas a reduction in skin temperature alone causes no effect [16]. In addition, norepinephrine and epinephrine responses to exercise were unchanged or marginally changed by mild body cooling, as compared those in a with neutral environment [5]. Our results suggest that mild body cooling with a decrease in skin temperature of approximately $2^{\circ} \mathrm{C}$ does not affect substrate oxidation during exercise or hormonal responses to exercise.

Although our hypotheses were mostly supported, there are several limitations in the present study. First, we examined a small sample size, and all participants were healthy and medication-free young men. Therefore, our results cannot be generalized to women, overweight/obese individuals, or elderly populations. Second, we did not measure skin blood flow and cardiac output at rest or during exercise. The mechanisms underlying the reduction in heart rate by mild body cooling should be investigated using these measurements. Finally, we examined the effects of mild body cooling in an environmentally controlled laboratory. Thus, future studies using the actual situations of climatic terrain therapy should be investigated.

\section{Conclusion}

Heart rate and subjective fatigue during exercise were reduced by mild body cooling, as compared with 
that during control conditions. However, a mild decrease in skin temperature did not affect fat and $\mathrm{CHO}$ oxidation or hormonal responses. Data on the reduction in heart rate and subjective fatigue during exercise observed in the mild body cooling trial may be useful when controlling exercise intensity for training or exercise prescriptions.

\section{References}

[1] Schuh, A. 1991. "Endurance Training under Simultaneous Cold Adaptation: Effects on Muscle Metabolism." Phys. Rehab. Kur. Med. 1: 22-8. (in German)

[2] Schuh, A. 1993. "Climatotherapy." Experientia 49 (11): 947-56.

[3] Gagnon, D. D., Rintamaki, H., Gagnon, S. S., Cheung, S. S., Herzig, K. H., Porvari, K., and Kyrolainen, H. 2013. "Cold Exposure Enhances Fat Utilization but Not Non-Esterified Fatty Acids, Glycerol or Catecholamines Availability during Submaximal Walking and Running." Frontiers in Physiology 4: 99.

[4] Weller, A. S., Millard, C. E., Stroud, M. A., Greenhaff, P. L., and Macdonald, I. A. 1997. "Physiological Responses to a Cold, Wet, and Windy Environment During Prolonged Intermittent Walking." The American Journal of Physiology 272 (1 Pt 2): R226-33.

[5] Layden, J. D., Patterson, M. J., and Nimmo, M. A. 2002. "Effects of Reduced Ambient Temperature on Fat Utilization during Submaximal Exercise." Medicine \& Science in Sports \& Exercise 34 (5): 774-9.

[6] Gagnon, D. D., Gagnon, S. S., Rintamaki, H., Tormakangas, T., Puukka, K., Herzig, K. H., and Kyrolainen, H. 2014. "The Effects of Cold Exposure on Leukocytes, Hormones and Cytokines during Acute Exercise in Humans." PLoS One 9 (10): e110774.

[7] Bogerd, N., Perret, C., Bogerd, C. P., Rossi, R. M., and Daanen, H. A. 2010. "The Effect of Pre-Cooling Intensity on Cooling Efficiency and Exercise Performance." Journal of Sports Sciences 28 (7): 771-9.

[8] Achten, J., and Jeukendrup, A. E. 2003. "Heart Rate Monitoring: Applications and Limitations." Sports Medicine 33 (7): 517-38.

[9] Bruce, R. A., Blackmon, J. R., Jones, J. W., and Strait, G. 1963. "Exercising Testing in Adult Normal Subjects and Cardiac Patients." Pediatrics 32 (SUPPL): 742-56.

[10] Tanaka, K., Takeshima, N., Kato, T., Niihata, S., and Ueda, K. 1990. "Critical Determinants of Endurance Performance in Middle-Aged and Elderly Endurance Runners with Heterogeneous Training Habits." European Journal of Applied Physiology and Occupational Physiology 59 (6): 443-9.

[11] Frayn, K. N. 1983. "Calculation of Substrate Oxidation Rates in Vivo from Gaseous Exchange." Journal of Applied Physiology: Respiratory, Environmental and Exercise Physiology 55 (2): 628-34.

[12] Ramanathan, N. L. 1964. "A New Weighting System for Mean Surface Temperature of the Human Body." Journal of Applied Physiology 19 (3): 531-3.

[13] Yeung, S. S., Ting, K. H., Hon, M., Fung, N. Y., Choi, M. M., Cheng, J. C., and Yeung, E. W. 2016. "Effects of Cold Water Immersion on Muscle Oxygenation During Repeated Bouts of Fatiguing Exercise: A Randomized Controlled Study.” Medicine (Baltimore) 95 (1): e2455.

[14] Williams, P. A., and Kilgour, R. D. 1993. "Cardiovascular Responses to Facial Cooling during Low and Moderate Intensity Exercise." European Journal of Applied Physiology and Occupational Physiology 67 (1): 53-8.

[15] Maw, G. J., Boutcher, S. H., and Taylor, N. A. 1993. "Ratings of Perceived Exertion and Affect in Hot and Cool Environments." European Journal of Applied Physiology and Occupational Physiology 67 (2): 174-9.

[16] Hurley, B. F., and Haymes, E. M. 1982. "The Effects of Rest and Exercise in the Cold on Substrate Mobilization and Utilization." Aviation, Space, and Environmental Medicine 53 (12): 1193-7. 